\title{
Stop, look, and listen when your patients 'complain'
}

A lead editorial in a recent issue of The New England Journal of Medicine (324;27:12061207) calls attention to the recently recognized postpolio syndrome. Although this syndrome reportedly existed more than 100 years ago, little or no attention has been paid to it since then.

The purpose of that editorial - as well as the one you are reading -is not to review in any significant detail the signs and symptoms of this syndrome. It seems sufficient to point out that postpoliomyelitis syndrome appears without any apparent antecedent. The patient begins to have a new level of fatigue, muscle weakness increases, muscle bulk diminishes, and sometimes, an unusual pain pattern develops. These symptoms occur most freqeuntly in those previously affected regions.

In writing his editorial, Theodore L. Munsat, MD, makes some casual observations that go far beyond the syndrome itself. "Credit for the recognition and definition of the postpolio syndrome must properly go to the many patients whose persistence eventually convinced their doctors that they were indeed experiencing new difficulties." Dr Munsat notes that most physicians apparently attributed the aforementioned symptoms to psychogenic or nonspecific impairment. Patients who first reported these symptoms to their physicians were not taken seriously. Only after these patients refused to be dismissed did the healthcare community begin to look at the problem more carefully.

Although both physicians and patients like to have their diseases "fit" in neat little verbal packages, many signs and symptoms are difficult to classify. Of course, too, we know that all patients are not equally lovable, nor do their diseases exemplify textbook descriptions. Unfortunately, we physicians often fail to give proper attention to those patients who come to our offices with complaints of unbelievable signs and symptoms. These patients can get sick and die of undiagnosed causes.

In earlier days, railroad crossings had signs telling the approaching motorist to "Stop, look, and listen" before advancing across the tracks. As we study our patients, we must stop long enough to perceive what they are saying and then give this information our due attention.

We need to look at the whole patient as a body unit, and we need to listen to the patient. Someone once said that patients will tell you their diagnosis if you can only give them the time and help them with the description. We need to know how to listen as much as we need to talk. Actually, it is difficult to listen if one is doing all the talking.

It's easy to say that bizarre symptoms are "stress-related." Stress has become the modern medical scrap bag into which we habitually throw some weird, different physical and mental conditions. This postpolio syndrome serves as a splendid example of how some physical signs, which we don't necessarily understand, may turn out to have meaningful ramifications. These ramifications may unveil many medical truths.

A good physician stops long enough to observe his or her patients both as a physician would and as someone who is a member of the healthcare community. We need to look with more than a casual eye at those physical signs or symptoms our patients bring to our attention. Sometimes, patients have difficulty expressing themselves, and it's not always easy for us to decode their message.

Most of all, we physicans need to listen to our patients and establish a credible line of communication. In the case of the postpolio syndrome, patients persevered until some physi- 
cians finally awakened to their patients' complaints; thus, an important medical observation was made.

The railroad signals-Stop, look, and listen-are excellent bywords for every physician to practice. They can admonish us of that oncoming "train"-illness or disease.

GEORGE W. NORTHUP, DO

Editor Emeritus

\section{PEF flow meter plays important role in asthma therapy}

Asthma, a hyperreactive airway disease, is characterized by inflammation, excessive mucus secretion, and bronchospasm that results in intermittent episodes of respiratory distress. Medication such as $\beta$-agonists, theophylline, and anti-inflammatory therapy-inhaled or systemic corticosteroids - can reverse this obstruction if it does not subside spontaneously.

Generally, asthma occurs at specific times during the day. Approximately $70 \%$ of patients have asthma-related sleep disturbance at least once a week even when they comply with their therapeutic regimen.

As the incidence, severity, and mortality of asthma appear to be on the rise, physicians are constantly searching for better ways of monitoring this disease. Primary care physicians, pulmonologists, and allergists recommend that patients use an inexpensive peak expiratory flow (PEF) meter to assess their own airway status. Lightweight and easy-touse, although somewhat bulky, the PEF meter affords an excellent means of evaluating pediatric or adult patients' airflow in the hospital or, more importantly, on an outpatient basis.

Patients require some minor instruction on how to use the device effectively. Additionally, they should be taught how to use metered- dose inhalers correctly, which are commonly used to deliver medication. Either the physician or a member of the office staff can instruct patients during their office visit. Patients should be told to use the PEF meter when they stand or sit upright. After taking a deep breath, patients should blow out as forcibly as possible in the shortest time. A nose clip is not necessary.

After each use, the device must be reset to zero. Patients should record the date and the highest numeric value of three consecutive PEF measurements in a notebook each time they use the device. In this way, diurnal and nocturnal asthma symptoms can be correlated with an actual flow measurement. Both the patient and the physician will better appreciate asthma's temporal dependencies.

Because asthma typically occurs or worsens at night, PEF has a rather prominent circadian rhythm. Persons who are active during the day and sleep at night tend to have their best PEF measurement around midday, while their worst PEF measurement occurs in the early morning hours, around $4 \mathrm{AM}$. Conversely, the asthmatic individual who works nights, for example, and sleeps during the day generally experiences difficulty breathing during daytime sleep.

Persons with normal pulmonary function have a high mean 24-hour airflow within the range of predicted values for persons of corresponding age and body build. Moreover, the day-night variability in airflow remains minimal, with generally no more than $10 \%$ of the 24-hour mean level.

Among asthma patients, however, the mean daily PEF is often reduced considerably from that of normal peak reference values; the circadian variability is enhanced. Generally, the daily mean PEF declines and circadian variability increases as the airways become unstable with a worsening of the disease.

(continued on page 860 ) 